\title{
Westward Spread of Highly Pathogenic Avian Influenza A(H7N9) Virus among Humans, China
}

\author{
Qiqi Yang, ${ }^{1}$ Wei Shi, ${ }^{1}$ Lei Zhang, ${ }^{1}$ Yi Xu, \\ Jing $\mathrm{Xu}$, Shen Li, Junjun Zhang, Kan Hu, \\ Chaofeng Ma, Xiang Zhao, Xiyan Li, Feng Liu, \\ Xin Tong, Guogang Zhang, Pengbo Yu, \\ Oliver G. Pybus, Huaiyu Tian
}

We report infection of humans with highly pathogenic avian influenza A(H7N9) virus in Shaanxi, China, in May 2017. We obtained complete genomes for samples from 5 patients and from live poultry markets or farms in 4 cities. Results indicate that H7N9 is spreading westward from southern and eastern China.

\begin{abstract}
A vian influenza $\mathrm{A}(\mathrm{H} 7 \mathrm{~N} 9)$ virus caused 5 waves of human infection in China from its emergence in 2013 (1) through May 17, 2017. During that period, 1,564 laboratory-confirmed human cases and 612 deaths were reported; about half occurred during the fifth wave (2). The fifth wave not only infected many more persons but also spread north; previous H7N9 outbreaks had been documented only in eastern and southern China. Moreover, viruses recently isolated from human case-patients in Guangdong Province (A/Guangdong/17SF003/2016 and A/ Guangdong/17SF006/2017) in southern China have been confirmed as highly pathogenic avian influenza (HPAI) $\mathrm{A}(\mathrm{H} 7 \mathrm{~N} 9)$ viruses, on the basis of their molecular (3) and biologic (4) characteristics.

We report cases of human infection with H7N9 virus, including in 1 person who was infected with a highly pathogenic variant, in Shaanxi Province, western China, during April and May 2017. We obtained complete genome sequences of H7N9 viruses from 5 patients and from 21 environmental samples obtained from live poultry markets (LPMs) and from poultry farms in 4 cities.
\end{abstract}

Author affiliations: Beijing Normal University, Beijing, China (Q. Yang, X. Tong, H. Tian); Shaanxi Provincial Centre for Disease Control and Prevention, Xi'an, China (W. Shi, L. Zhang, Y. Xu,

J. Xu, S. Li, F. Liu, P. Yu); Xianyang Centre for Disease Control and Prevention, Xianyang, China (J. Zhang); Baoji Centre for Disease Control and Prevention, Baoji, China (K. Hu); Xi'an Centre for Disease Control and Prevention, X'an (C. Ma); Chinese Center for Disease Control and Prevention, Beijing (X. Zhao, X. Li); Chinese Academy of Forestry, Beijing (G. Zhang); University of Oxford, Oxford, UK (O.G. Pybus)

\section{The Study}

A case of HPAI H7N9 virus infection in a human from Shaanxi Province was identified in the city of Yulin on May 26, 2017. On day 1 of illness onset, fever and fatigue developed; after 2 days, the patient received medical attention. On day 4, the patient was admitted to an intensive care unit, and on day 6, HPAI H7N9 infection was laboratory confirmed. After treatment with oseltamivir, the patient recovered. An additional 4 cases of infection with low pathogenicity avian influenza (LPAI) A(H7N9) virus among humans were identified during April 30-May 13, 2017, in Shaanxi Province: 2 in Xianyang, 1 in Baoji, and 1 in the capital city, Xi' an. Recorded symptoms included fever (100\%); cough and fatigue (75\%); and productive cough, diarrhea, and shortness of breath $(50 \%)$. Patients received medical attention at an average of 3.75 (range 1-5) days after illness onset and were admitted to a hospital at an average of 6 (range 4-8) days after onset. Samples were laboratory confirmed as H7N9 virus at an average of 9 (range 6-14) days after onset. Of the 5 patients, 4 were treated with oseltamivir; 2 of those died.

Respiratory tract specimens (throat swab or sputum sample) were collected from patients and transferred to the national laboratory at the Chinese Center for Disease Control and Prevention (China CDC) for confirmation. Viral RNA was extracted from each sample by using nucleic acid isolation magnetic beads, according to the manufacturer's instructions (TianLong Science and Technology Co. Ltd. Xi' an, China). Avian influenza virus $\mathrm{H} 7$ and N9 gene segments were detected in the total RNA by using an H7N9 dual-channel Taqman probe reverse transcription PCR kit (BioPerfectus Co. Ltd. Taizhou, China).

During May 2017, we collected 496 environmental samples (poultry feces and poultry cage swabs from LPMs and poultry farms) from 4 cities (Xi'an, Baoji, Xianyang, and Yuli) in Shaanxi Province (online Technical Appendix Table 1, https://wwwnc.cdc.gov/EID/article/24/6/17-1135Techapp1.pdf). A total of 14 samples from LPMs were positive for H7N9 virus.

We extracted total RNA from the patient respiratory specimens and from H7N9-positive environmental samples and sequenced avian influenza virus genomes by using next-generation sequencing on an Illumina MiSeq (Illumina Inc., Shanghai, China). Sequence comparisons

${ }^{1}$ These authors contributed equally to this article. 
showed that the viruses we isolated from 1 patient in Yulin and from the 15 environmental samples had the same polybasic (PB) amino acid sequence (PEVPKRKRTAR/GL) at the hemagglutinin (HA) cleavage site as that in previously confirmed HPAI H7N9 viruses (A/Guangdong/17SF003, A/Guangdong/17SF006, and A/Taiwan/1/2017) (3-5), indicating that the viruses we isolated are also HPAI viruses. In addition, the HPAI viruses we isolated appear to have inherited the HA amino acid mutation G186V from LPAI viruses and reverted to $226 \mathrm{Q}$ from $226 \mathrm{~L}$, as observed in previously confirmed HPAI H7N9 viruses (4-6). Because G186V and Q226L indicate an increased level of binding to human-type influenza receptors $(7,8)$, the ability of the HPAI viruses we isolated here to infect humans is likely similar to that of previously reported HPAI H7N9 viruses. Furthermore, the HA genes of all Shaanxi HPAI H7N9 viruses we isolated were $>99 \%$ identical to those of previously isolated HPAI H7N9 viruses.
We analyzed each of 8 gene segments of H7N9 viruses by using phylogenetic methods, together with available sequences from the GISAID EpiFlu database (http:// platform.gisaid.org/; accession numbers in online Technical Appendix Table 3). The HA and neuraminidase (NA) phylogenies indicate that the viruses isolated in Shaanxi belong to clade W2-C (9). In the HA tree (Figure 1; online Technical Appendix Figure 1), the 16 HPAI H7N9 viruses we isolated clustered with previously detected HPAI H7N9 viruses from Guangdong Province. The HA gene of the Shaanxi HPAI H7N9 viruses has a common ancestor with the Guangdong HPAI H7N9 viruses, indicating that HPAI H7N9 viruses have spread from Guangdong to other provinces (online Technical Appendix Figure 2, panel B). Further, the 7 LPAI H7N9 viruses we identified in Shaanxi Province clustered with LPAI H7N9 viruses isolated from various districts in eastern (Anhui, Fujian, Zhejiang, Jiangsu), central (Chongqing, Henan, Hunan, Hubei), and

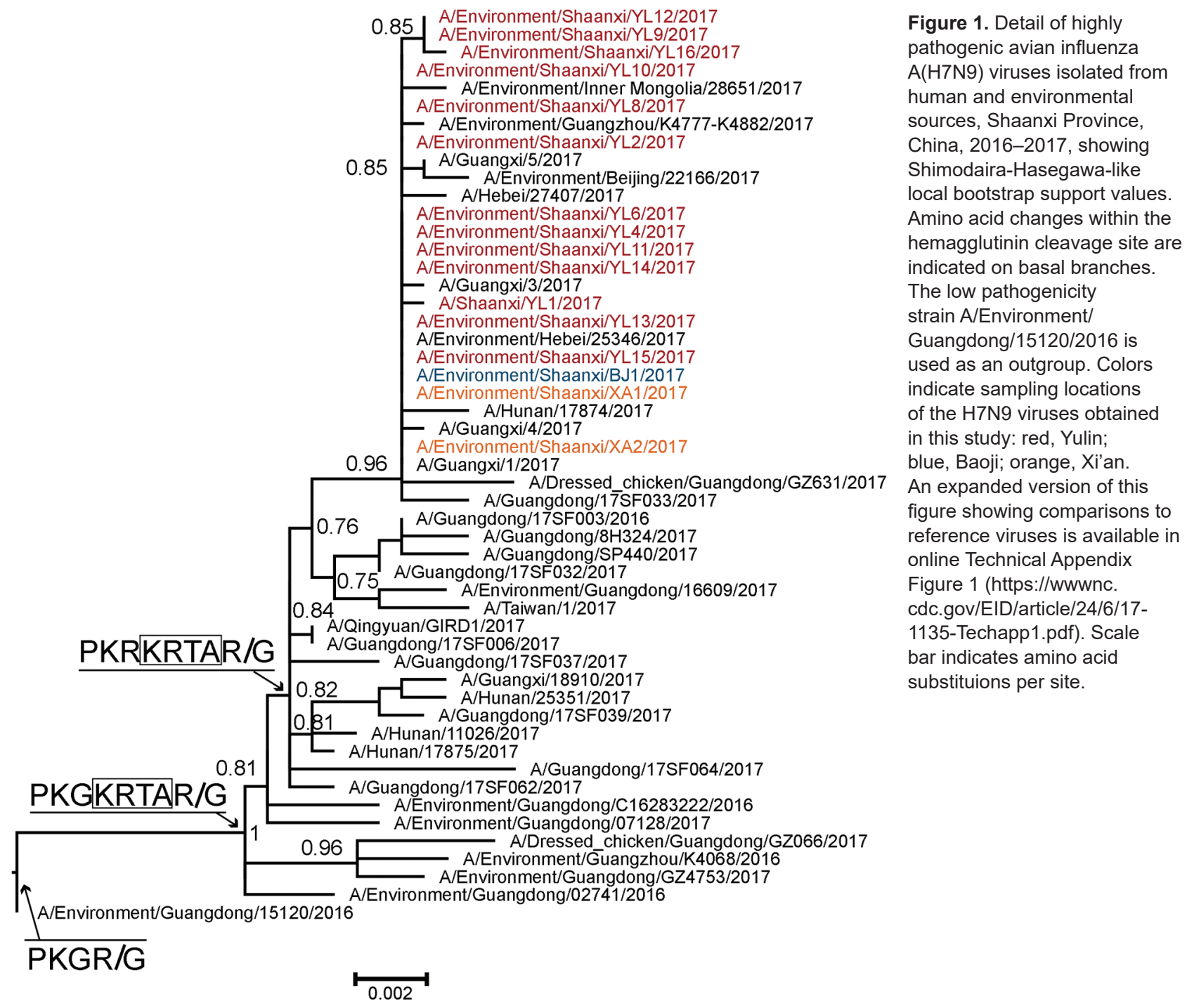


southern (Guangxi, Guangdong, Hong Kong) China, indicating that LPAI H7N9 viruses have been spreading from eastern and southern China into western China (Figure 2). In the NA phylogeny, the phylogenetic positions of the NA genes of all viruses we sequenced were similar to those observed in the HA phylogeny (online Technical Appendix Figure 2). Phylogenies of the 6 internal genes (online Technical Appendix Figure 3) show that the viruses from Shaanxi Province belong to clades 1 and 2 (9) of the ZJ$\mathrm{HJ} / 07$ lineage of H9N2 viruses (10) (online Technical Appendix Table 2).

Phylogenetic analyses of HA and NA gene segments reveal that the viruses obtained from human cases in Yulin and Baoji were genetically similar to those from environmental samples collected from local farms or LPMs in Yulin and Baoji (online Technical Appendix Figure 1, panel A; online Technical Appendix Figure 2), suggesting that the human H7N9 infections are related to viruses circulating in local farms or LPMs. This hypothesis is also supported by most internal gene segments, except for the NS segments of viruses from Yulin (no internal gene segments were obtained for isolate A/Environment/Shaanxi/ BJ2/2017 sampled in Baoji). Most of the gene segments of the viruses from human cases in Baoji and Xianyang were similar to those of A/Environment/Shaanxi/XA4/2017, the virus isolated from LPMs in Xi'an (with the exception of PB1 from the human case in Baoji and PB2, PB1, and matrix of the viruses from human cases in Xianyang). The NS and polymerase acidic segments of the LPAI virus from a human case in Xi'an (A/Shaanxi/XA1/2017) were genetically similar to the HPAI viruses we isolated; other gene segments were close to LPAI viruses from Anhui Province in southern China.

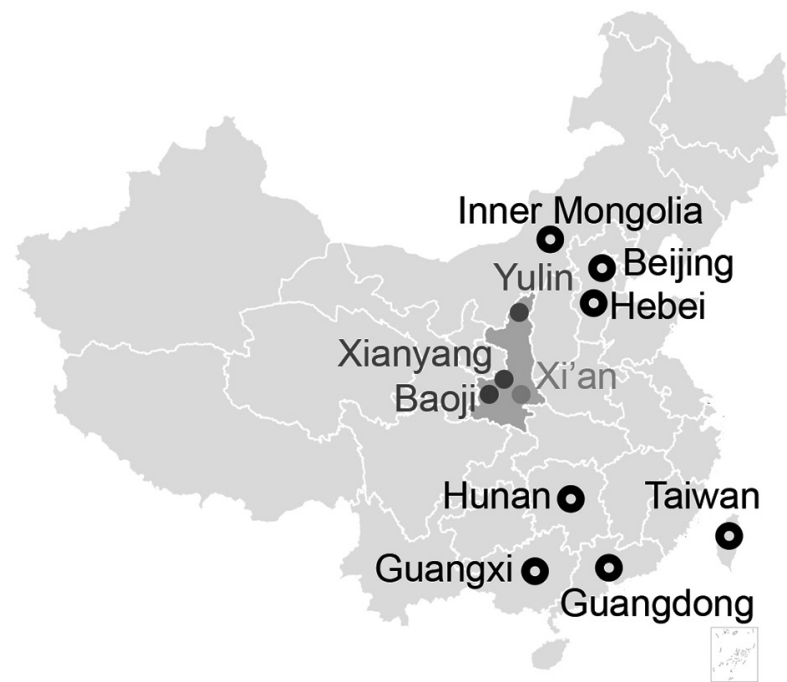

Figure 2. Geographic distribution of the avian influenza $A(H 7 N 9)$ viruses isolated in Shaanxi Province, China, 2016-2017 (solid circles), and of HPAI H7N9 viruses detected in other provinces of China (open circles).

\section{Conclusions}

We report emerging HPAI H7N9 variants in Shaanxi Province, western China. Our phylogenetic analyses support that the LPAI H7N9 viruses in Shaanxi Province originated from eastern and southern China, and the Shaanxi HPAI H7N9 isolates probably originated in Guangdong Province and were transmitted either directly or indirectly through other provinces.

Our results provide evidence that H7N9 viruses infecting humans in Shaanxi Province derived, directly or indirectly, from strains circulating in local farms and LPMs. Previous studies suggest that poultry trade between LPMs may play a key role in spreading H7N9 viruses (11). Similarly, we hypothesize that live poultry or poultry product transport may facilitate the spread of HPAI H7N9 viruses out of Guangdong Province and within Shaanxi Province.

\section{Acknowledgments}

We thank Katie Oakley for her valuable comments and help.

This work was supported by the National Natural Science Foundation of China (81673234); National Key Research and Development Program of China (2016YFA0600104); the Fundamental Research Funds for the Central Universities; Health Industry's Special Research Funds for Public Welfare Projects (201502020); Shaanxi Provincial Projects for Serious Disease Prevention and Control (2014A7); and Science and Technology Project of Shaanxi Province (2016E010, 2016D092, 2016D095; http://www.sxcdc.com/). The funding sources had no role in the study design, data collection and analysis, decision to publish, or preparation of the manuscript.

\section{About the Author}

Dr. Yang is a postgraduate student at State Key Laboratory of Remote Sensing Science, College of Global Change and Earth System Science, Beijing. Her research interests are phylogenetics and phylodynamics of influenza viruses.

\section{References}

1. Gao R, Cao B, Hu Y, Feng Z, Wang D, Hu W, et al. Human infection with a novel avian-origin influenza $\mathrm{A}(\mathrm{H} 7 \mathrm{~N} 9)$ virus. N Engl J Med. 2013;368:1888-97. http://dx.doi.org/10.1056/ NEJMoa1304459

2. World Health Organization. Monthly Risk Assessment Summary. Influenza at the human-animal interface. 2017 Oct 30 [cited 2017 Dec 7]. http://www.who.int/influenza/human_animal_interface/ Influenza_Summary_IRA_HA interface_10 30 2017.pdf

3. Zhang F, Bi Y, Wang J, Wong $\bar{G}$, Shi W, $\bar{H} u \bar{F}$, et al. Human infections with recently-emerging highly pathogenic H7N9 avian influenza virus in China. J Infect. 2017;75:71-5. http://dx.doi.org/10.1016/j.jinf.2017.04.001

4. Zhu W, Zhou J, Li Z, Yang L, Li X, Huang W, et al. Biological characterisation of the emerged highly pathogenic avian influenza (HPAI) A(H7N9) viruses in humans, in mainland China, 2016 to 2017. Euro Surveill. 2017;22:30533. http://doi.org/10.2807/ 1560-7917.ES.2017.22.19.30533 
5. Yang JR, Liu MT. Human infection caused by an avian influenza A (H7N9) virus with a polybasic cleavage site in Taiwan, 2017. J Formos Med Assoc. 2017:116(3):210-2. http://doi.org/10.1016/ j.jfma.2017.02.011

6. Changwen K, Chris Ka Pun M, Wenfei Z, Haibo Z, Jianfeng H, Wenda $G$, et al. Human infection with highly pathogenic avian influenza A(H7N9) virus, China. Emerging Infect Dis J. 2017;23:1332-1340. http://dx.doi.org/10.3201/eid2308.170600

7. Shi Y, Zhang W, Wang F, Qi J, Wu Y, Song H, et al. Structures and receptor binding of hemagglutinins from human-infecting H7N9 influenza viruses. Science. 2013;342:243-7. http://dx.doi.org/ 10.1126/science. 1242917

8. Zhou J, Wang D, Gao R, Zhao B, Song J, Qi X, et al. Biological features of novel avian influenza A (H7N9) virus. Nature. 2013;499:500-3. http://dx.doi.org/10.1038/nature12379

9. Lam TT, Zhou B, Wang J, Chai Y, Shen Y, Chen X, et al. Dissemination, divergence and establishment of H7N9 influenza viruses in China. Nature. 2015;522(7554):102-5. http://dx.doi.org/ 10.1038 /nature 14348

10. Lam TT, Wang J, Shen Y, Zhou B, Duan L, Cheung C-L, et al. The genesis and source of the H7N9 influenza viruses causing human infections in China. Nature. 2013;502(7470):241-4.

11. Zhou X, Li Y, Wang Y, Edwards J, Guo F, Clements ACA, et al. The role of live poultry movement and live bird market biosecurity in the epidemiology of influenza A (H7N9): a cross-sectional observational study in four eastern China provinces. J. Infect. 2015Oct;71(4):470-9. http://dx.doi.org/ 10.1016/j.inf.2015.06.012

Address for correspondence: Huaiyu Tian, State Key Laboratory of Remote Sensing Science, College of Global Change and Earth System Science, Beijing Normal University, Beijing 100875, China; email: tianhuaiyu@gmail.com

\section{December 2013: Zoonotic Infections}

- Institute of Medicine and National Research Council Recommendations for One Health Initiative

- Epidemiologic Investigations into Outbreaks of Rift Valley Fever in Humans, South Africa, 2008-2011

- Potential Role of Deer Tick Virus in Powassan Encephalitis Cases in Lyme Disease-endemic Areas of New York, USA
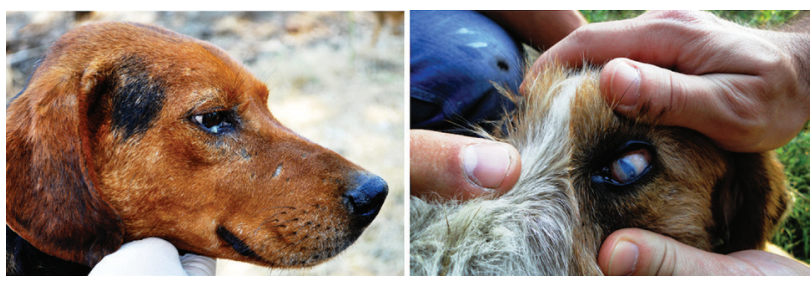

- Twenty-Year Summary of Surveillance for Human Hantavirus Infections, United States

- Spontaneous Generation of Infectious Prion Disease in Transgenic Mice

- Zoonotic Chlamydiaceae Species Associated with Trachoma, Nepal

- Guillain-Barré Syndrome Surveillance during National Influenza Vaccination Campaign, New York, USA, 2009

- Antiviral Susceptibility of Highly Pathogenic Avian Influenza $A(\mathrm{H} 5 \mathrm{~N} 1)$ Viruses Isolated from Poultry, Vietnam, 2009-2011

- Novel Reassortant Influenza A(H1N2) Virus Derived from $A(H 1 N 1) p d m 09$ Virus Isolated from Swine, Japan, 2012

- Myocarditis after Trimethoprim/Sulfamethoxazole Treatment for Ehrlichiosis

- Distinct Lineage of Vesiculovirus from Big Brown Bats, United States
- Novel Cause of Tuberculosis in Meerkats, South Africa

- Acute Toxoplasma gondii Infection among Family Members in the United States

- Novel Orthoreovirus from Mink, China, 2011

- Outbreak of Human Infection with Sarcocystis nesbitti, Malaysia, 2012

- Transmission of Brucellosis from Elk to Cattle and Bison, Greater Yellowstone Area, USA, 2002-2012

- Concomitant Human Infections with 2 Cowpox Virus Strains in Related Cases, France, 2011

- Zoonotic Onchocerca lupi infection in Dogs, Greece and Portugal, 2011-2012

- Cerebellar Cysticercosis Caused by Larval Taenia crassiceps Tapeworm in Immunocompetent Woman, Germany

- Powassan Virus in Mammals, Alaska and New Mexico, USA, and Russia, 2004-2007

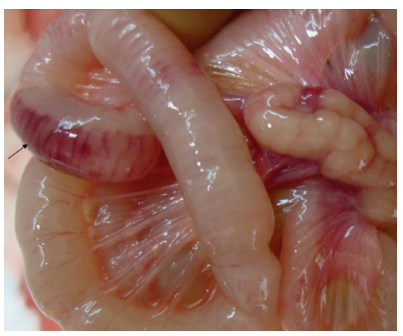

- Reemergence of Vaccinia Virus during Zoonotic Outbreak, Pará State, Brazil

- Historical Prevalence and Distribution of Avian Influenza Virus $\mathrm{A}(\mathrm{H} 7 \mathrm{~N} 9)$ among Wild Birds

- Lack of MERS Coronavirus Neutralizing Antibodies in Humans, Eastern Province, Saudi Arabia

- Peste des Petits Ruminants Infection among Cattle and Wildlife in Northern Tanzania

- Surveillance for Avian Influenza A(H7N9), Beijing, China, 2013 